\title{
Carnets
}

Revue électronique d'études françaises de l'APEF

Deuxième série - 4 | 2015

Regards sur Camus

\section{« Solitaire ou solidaire » dans L'Exil et le Royaume d'Albert Camus}

\section{Fernando Gomes}

\section{Q OpenEdition}

1 Journals

\section{Édition électronique}

URL : http://journals.openedition.org/carnets/1559

DOI : 10.4000/carnets.1559

ISSN : 1646-7698

Éditeur

APEF

\section{Référence électronique}

Fernando Gomes, « «Solitaire ou solidaire » dans L'Exil et le Royaume d'Albert Camus », Carnets [En ligne], Deuxième série - 4 | 2015, mis en ligne le 30 mai 2015, consulté le 30 avril 2019. URL : http:// journals.openedition.org/carnets/1559; DOI : 10.4000/carnets.1559

Ce document a été généré automatiquement le 30 avril 2019.

\section{(c) (i) (8)}

Carnets est mis à disposition selon les termes de la licence Creative Commons - Atribution - Pas d'utilisation commerciale 4.0 International. 


\title{
« Solitaire ou solidaire » dans L'Exil et le Royaume d'Albert Camus
}

\author{
Fernando Gomes
}

La solidarité est indubitablement la solution préconisée par Albert Camus pour faire face à la crise humanitaire engendrée par les atrocités de La Deuxième Guerre mondiale. Dans La Peste et L'Homme révolté, il cherche à faire de cette pensée une valeur morale qui, en l'absence de Dieu, unirait les hommes et les défendrait des maux de la société. Toutefois, n'existant que comme une alliance face à l'adversité, sa conception de solidarité s'avère instable. Ainsi, pour André Nicolas, dans La Peste, il n'existerait qu'une sorte de sympathie obscure entre les personnages, que leur solidarité ne serait qu'une "vague fraternité » (Nicolas, 1964: 178). Brian T. Fitch renforce cette idée, soulignant qu'il y a souvent un contact physique entre les individus mais que « le monde de Camus reste essentiellement un univers egocentrique et solitaire» (Fitch, $1964: 217)$. Toutefois, dans son adaptation de Requiem de William Faulkner, Camus fait dire à Temple Stevens que, elle et Nancy sont " solitaires ensemble ", ce qui nous semble être un pas vers la fraternité, ne serait-ce que dans la solitude, thème essentiel des nouvelles réunies dans le recueil L'Exil et le Royaume.

2 Camus, plus que nulle part ailleurs, y évoque la complexité des relations humaines, mélange de solidarité, parfois fraternelle, de silence et de solitude. Cette complexité de sentiments émane de l'état d'esprit de Camus face à son exil, à la crise algérienne et à ses doutes identitaires. Roger Quilliot résume ainsi cette période de la vie de Camus :

Tout d'abord, l'atmosphère de solitude, d'exil, dans laquelle il entreprend cette troisième partie de son œuvre : exil parisien, solitude de l'écrivain devenu trop célèbre et du même coup accablé de corvées, jalousé, souvent; sentiment douloureux d'être prisonnier de ses œuvres, de son vocabulaire, d'une certaine image inexacte que la critique et le public ont composé de lui. (Quilliot, $2002: 2038$ ) Non seulement il s'interroge sur la valeur et le sens de son œuvre, non seulement il se découvre envié, contesté, sans bien comprendre pourquoi, mais il en vient à douter du sens de son action et de son propre visage. (Quilliot, $2002: 2040$ )

Tout entier prisonnier de la littérature et des littérateurs, [il a] cette angoisse de l'homme prisonnier de sa légende et de son métier. (Quilliot, $2002: 2053$ ) 
La Chute, qui devait intégrer L'Exil et le Royaume, témoigne de cette crise intérieure et constitue, par là-même, «la meilleure introduction» (Grouix, 2009: 302) à ce recueil. C'est, en effet, pendant cette période, ou plus précisément, de 1952 à 1954, à la veille de la l'explosion de la guerre d'Algérie, qu'est né le projet de ces nouvelles : trois se déroulent en Algérie - «La Femme adultère ", "Le Renégat ou un esprit confus ", "Les Muets ", «L'Hôte »-, une à Paris « Jonas ou l'Artiste au travail » et l'autre sur la relation européenautochtone dans le contexte du Brésil «La Pierre qui pousse ». Polyphoniques, elles ont en commun l'exil, psychologique et/ou géographique, et la quête du royaume, dévoilant par là même diverses facettes de la relation de leur auteur avec ses contemporains et avec son pays natal, l'Algérie.

4 Bien que «La Femme adultère » ouvre ce recueil, nous commencerons notre étude centrée sur la thématique de la solitude et la solidarité - avec une lecture de la cinquième nouvelle «Jonas ou l'artiste au travail », pour deux raisons : la première parce qu'elle nous fournit les deux mots clés à notre étude - solitaire et solidaire (Camus, 2002 : 1654) et la deuxième parce que la transition progressive de l'ironie, qui établit une distance entre l'auteur et son personnage, vers un ton plus sérieux aboutissant au drame final laisse deviner des affinités plus profondes entre Camus et Jonas. Ainsi, cette nouvelle met en scène un artiste peintre un peu candide, victime d'un grand succès qu'il attribue à son étoile. En effet, son espace vital est progressivement envahi par des admirateurs qui se disent ses amis - "Ce fut le moment aussi où le succès de Jonas lui valut beaucoup d'amis» (Camus, 2002: 1634) -, et par des disciples, bref par tout un éventail mondain, qui lui ôte toute vie privée. Prisonnier de cette multitude, sa solitude augmente alors que diminue sa production artistique, jusqu'au moment où il ne réussit plus à produire. Cette dégradation s'accompagne d'une réflexion intérieure et si, pendant un temps il cherche dans l'isolement, l'alcool et les femmes une échappatoire à cette crise, il pense trouver une solution à son problème en construisant une soupente dans son appartement qui, pendant qu'il peindrait, l'isolerait des autres hommes sans toutefois s'éloigner d'eux. Cherchant « ce secret qui n'était pas seulement celui de l'art » (Camus, $2002: 1652$ ) il va se reclure de plus en plus et, finalement, éviter tout contact avec les autres qui, à part son ami Rateau, finissent par ne plus fréquenter sa maison. C'est dans cet isolement presque total qu'il prend conscience du besoin qu'il a des autres et il en est heureux (Camus, 2002 : 1654). Bien qu'ayant pris la décision de ne plus travailler, les bruits de la rue et surtout ceux, plus familiers, de sa femme et de ses enfants, signes de la présence des autres, semblent réveiller en lui son étoile. Épuisé, il s'évanouit, mais on peut supposer qu'il aura retrouvé son inspiration. Reste le fruit de son isolement, de ses réflexions: "Rateau regardait la toile, entièrement blanche, au centre de laquelle Jonas avait seulement écrit, en très petits caractères, un mot qu'on pouvait déchiffrer, mais dont on ne savait s'il fallait y lire solitaire ou solidaire» (Camus, 2002: 1654). De cette crise intellectuelle et artistique, on pourrait conclure que, dans la perspective de Camus, il ne peut y avoir de solidarité sans solitude et que la solitude, comme source de création artistique, est intrinsèquement liée à la solidarité. Il est vrai que sans la présence des autres, Jonas ne parvient pas à créer. Dans son interprétation de la fin de cette nouvelle Raymond GayCrosier affirme :

(...) the inconclusiveness of the word he penned on his canvas constitutes the necessary catalyst for his artistic survival. The indeterminacy between solitaire and solidaire is not a lesson that Jonas teaches, it is message whose meaning he has yet to learn, to live, and to carry out when he descends from his loft and rejoins the world (Gay-Crosier, 1988 : 25). 
5 Jonas découvre qu'une œuvre d'art est aussi bien une invitation au silence qu'au partage, à la communion.

6 Le silence est également une des particularités de la relation du couple Janine et Marcel dans « La Femme adultère », silence qui expose toute la distance entre les époux. Au cours d'un voyage avec son mari dans l'arrière-pays, Janine confrontée à l'altérité géographique et humaine du désert, prend conscience du vide de sa vie quotidienne et de toute sa solitude. Du début à la fin de cette nouvelle, la seule mettant en scène une femme, Camus nous livre les pensées et les sentiments les plus intimes de son héroïne, ce qui constitue également une exception dans l'œuvre camusienne. Ainsi, nous apprenons qu'elle est profondément malheureuse face à son existence, à " cette vie démente et figée ", à la peur de vieillir et mourir seule, et particulièrement face à sa vie privée. De fait, la relation entre Janine et Marcel semble être dominée par des sentiments négatifs. Elle fait de son mari un portrait qui est loin d'être flatteur : «Elle avait fini par l'accepter, bien qu'il fût un peu petit et qu'elle n'aimât pas beaucoup son rire avide et bref, ni ses yeux noirs trop saillants »(Camus, 2002: 1560). Leur vie ensemble est résumée en des termes qui ne pourrait être plus pessimistes :

Sur la côte, les années de jeunesse peuvent être heureuses. Mais il n'aimait pas beaucoup l'effort physique et, très vite, il avait cessé de la mener sur les plages. (...) Ils n'avaient pas eu d'enfants. Les années avaient passé, dans la pénombre qu'ils entretenaient, volets mi-clos. L'été, les plages, les promenades, le ciel même étaient loin. Rien ne semblait intéresser Marcel que ses affaires. (...) Le plus dur était l'été où la chaleur tuait jusqu'à la douce sensation de l'ennui. (Camus, 2002 : 1561-62)

Il n'y a pas d'amour entre eux : " L'amour, même haineux, n'a pas ce visage renfrogné » et leurs rapport sexuels sont furtifs, empreints de honte : «Ils s'aimaient dans la nuit, sans se voir, à tâtons. Y a-t-il un autre amour que celui des ténèbres, un amour qui crierait en plein jour ? Elle ne savait pas, (...)» (Camus, 2002 : 1572). Janine a fini par tout devoir à Marcel car elle a un besoin vital de se sentir aimée : «Surtout, elle aimait être aimée. (...) À lui faire sentir si souvent qu'elle existait pour lui, il lui faisait exister réellement. Non, elle n'était pas seule...»(Camus, 2002 : 1560-61). Elle est consciente du fait que leur relation est plus complexe que cela, que tous deux fuient la mort et la solitude :

(...) elle savait que Marcel avait besoin d'elle et qu'elle avait besoin de ce besoin, qu'elle en vivait la nuit et le jour, la nuit surtout, chaque nuit, où il ne voulait pas être seul, ni vieillir, ni mourir, avec cet air buté qu'il prenait et qu'elle reconnaissait parfois sur d'autres visages d'hommes, le seul air commun de ces fous qui se camouflent sous des airs de raison, jusqu'à ce que le délire les prenne et les jette désespérément vers un corps de femme pour y enfouir, sans désir, ce que la solitude et la nuit leur montrent d'effrayant.

(...) Non, il ne l'aimait pas, il avait peur de ce qui n'était pas elle, simplement, et elle et lui depuis longtemps auraient dû se séparer, et dormir seuls jusqu'à la fin. Mais qui peut dormir toujours seul?

Elle l'appela de tout son cœur. Elle aussi, après tout avait besoin de lui, de sa force, de ses petites manies, elle aussi avait peur de mourir. (Camus, $2002: 1572$ )

8 Il semble que tous deux se sont réfugiés dans un univers clos, dans une routine réconfortante, sans amour mais dans une communion face à l'ultime solitude qu'est la mort. Néanmoins, placée hors de cet univers familier, fragilisée par la fatigue du voyage et par la confrontation avec l'altérité de la population autochtone et de la région, Janine se sent malheureuse. Du haut de la terrasse du fort, face à l'espace sans fin, elle reste sans voix « silencieuse devant l'étendue sans limites » (Camus, 2002 : 1569). Janine aperçoit au 
loin un campement de nomades et, dans la solitude de la terrasse, elle expérimente son premier appel du désert :

Janine (...) restait sans voix, incapable de s'arracher au vide qui s'ouvrait devant elle. (...) Là-bas, plus au sud encore, à cet endroit où le ciel et la terre se rejoignaient dans une ligne pure, là-bas, lui semblait-il soudain, quelque chose l'attendait qu'elle avait ignoré jusqu'à ce jour et qui pourtant n'avait cessé de lui manquer. (Camus, $2002: 1569-70)$

9 Janine sent se réveiller en elle sa féminité, se dénouer en elle, « un nœud que les années, l'habitude et l'ennui avaient serré " (Camus, 2002: 1570). Elle sent naître en elle un profond désir de cette vie nomade :

Elle n'avait même pas vu les hommes qui vivaient là, rien ne bougeait (...) et, pourtant, elle ne pouvait penser qu'à eux, dont elle avait à peine connu l'existence jusqu'à ce jour. (...) Depuis toujours, sur la terre sèche, raclée jusqu'à l'os, de ce pays démesuré, quelques hommes cheminaient sans trêve, qui ne possédaient rien mais ne servaient personne, seigneurs misérables et libres d'un étrange royaume. (Camus, $2002:$ :1570)

10 Si dans un premier temps elle parle du désert comme d'un « royaume de pierre » (1569) ${ }^{1}$, symbole de la pétrification de sa vie avec Marcel, dans un second temps, se référant à « l'étrange royaume» (Camus, 2002: 1570), elle pense aussi bien au pays qu'au mode de vie des nomades, une vie de liberté qui semble vaguement répondre à des désirs issus de tout un imaginaire orientaliste ${ }^{2}$.

11 Au milieu de la nuit, elle se rend compte de son malheur et sent toute son impuissance face à la mort. Elle cherche alors à être « délivrée ». Répondant à l'appel de la nuit ${ }^{3}$, elle se lève et, sur la terrasse du fort, elle expérimente une communion mystique avec le ciel et le désert. Camus présente cette relation comme une union presque charnelle qui culmine en ce qui pourrait être perçu comme l'accouchement d'elle-même. Janine commet ainsi son adultère, lequel momentanément, lui redonne vie et lui permet d'accéder au royaume :

Après tant d'années où, fuyant devant la peur, elle avait couru follement, sans but, elle s'arrêtait enfin. En même temps, il lui semblait retrouver ses racines, la sève montait à nouveau dans son corps qui ne tremblait plus. Pressée de tout son ventre contre le parapet, tendue vers le ciel en mouvement, elle attendait seulement que son cœur encore bouleversé s'apaisât à son tour et que le silence se fit en elle. Les dernières étoiles des constellations laissèrent tomber leurs grappes un peu plus bas sur l'horizon du désert, et s'immobilisèrent. Alors, avec une douceur insupportable, l'eau de la nuit commença d'emplir Janine, submergea le froid, monta peu à peu du centre obscur de son être et déborda en flots ininterrompus jusqu'à sa bouche pleine de gémissements. L'instant d'après, le ciel entier s'étendait au-dessus d'elle, renversée sur la terre froide. (Camus, $2002: 1574-75)$

12 Janine retrouve ce royaume qu'elle avait connu autrefois et duquel elle s'était exilée; royaume du titre du recueil que Camus dans son «Prière d'insérer » définit ainsi : «Il coïncide avec une certaine vie libre et nue que nous avons à retrouver, pour renaître enfin. L'exil, à sa manière, nous en montre les chemins, à la seule condition que nous sachions y refuser en même temps la servitude et la possession » (Camus, 2002 : 2039).

13 L'existence de l'«autre " ${ }^{4}$, du nomade, symbole d'une vie libre, réveille en Janine des sentiments refoulés qui lui permettent de renaître et de retrouver ses racines. Cependant ce moment est passager et il n'y a pas de véritable échange avec l'« autre ». Dans ce sens, Fitch commente : 
When Janine turns away from Marcel, abandoning the conjugal bed, she does not turn to any human lover. In fact, it is no exaggeration at all to claim that in turning away from her husband, she turns away at the same time and by the same gesture from all other men. Her partner in her act of sexual infidelity is none other than the whole of nature and its elements. (Fitch, $1988: 122$ )

Bouleversée par cette expérience, Janine retourne à l'exil, au lit conjugal, à une solitude à deux : «Elle pleurait, de toutes ses larmes, sans pouvoir se retenir» (Camus, 2002 : 1575). Ces larmes ont été amplement interprétées, nommément para Peter Cryle qui, après avoir résumé les diverses critiques, essaye de répondre à la question : « Pourquoi Janine pleuret-elle?» (Cryle, 1973: 64). Ce sont des larmes de joie d'avoir accédé au royaume ou des larmes de tristesse d'être retournée à son exil ? Nous sommes ici face à une ambivalence typique de l'ironie camusienne.

Symbole d'une solitude régénératrice dans "La Femme adultère », le désert acquiert des caractéristiques négatives pour se transformer en lieu de violence et d'annihilation de l'individu dans « Le Renégat ou un esprit confus ».

Cette nouvelle évoque l'échec de la tentative de conversion d'une culture noneuropéenne aux valeurs sociales et morales occidentales, nommément au catholicisme. La confusion mentionnée dans le sous-titre ne se réfère pas seulement à l'évident manque de compréhension du narrateur en ce qui concerne la vraie essence des deux cultures entre lesquelles il se sent prisonnier. De fait, cette confusion expose aussi l'impossible communication entre deux cultures qui, dans un même espace sociopolitique, présentent des valeurs irréductibles.

17 Tout le texte, excepté la dernière phrase, est un monologue intérieur d'un missionnaire prisonnier d'une tribu du désert - « cette terre [qui] rend fou ", « à la frontière de la terre des noirs e du pays blanc » (Camus, $2002:$ 1579, 1582) -, dans une ville de sel, Taghâsa. Outre la possible castration, les indices de sauvagerie de la tribu et de leur insoumission au pouvoir colonial acquièrent un symbolisme particulier dans l'acte d'excision de la langue du missionnaire. Cette mutilation, signe de la violence du discours politique, pervertit de prime abord, l'image romantique du désert présente dans Noces, le transformant en lieu de violence qui nie l'amour et la fraternité et exalte le règne du mal et de la solitude. En vérité, dans cette nouvelle, Camus présente, en termes fictionnels, un discours inédit sur la violence des extrémismes de la crise Algérienne qu'il expose dans Actuelles III - Chroniques algériennes 1939-1958. Dans son "Avant-propos», Camus critique les interventions partidaires :

C'est pourquoi dans l'impossibilité de me joindre à aucun des camps extrêmes, (...) j'ai décidé de ne plus participer aux incessantes polémiques qui n'ont eu d'autre effet que de durcir en Algérie les intransigeances aux prises et de diviser un peu plus une France déjà empoisonnée par les haines et les sectes. (Camus, $2000: 891$ )

Camus décrit aussi l'Algérie comme une jungle où l'unique principe serait la violence (Camus, 2000 : 892-93). Plus avant, il condamne le recours à la violence de la part des deux factions :

La vérité hélas! C'est qu'une partie de notre opinion pense obscurément que les Arabes ont acquis le droit, d'une certaine manière, d'égorger et de mutiler tandis qu'une autre partie accepte de légitimer, d'une certaine manière, tous les excès. Chacun, pour se justifier, s'appuie alors sur le crime de l'autre. (Camus, $2000:$ : 895)

19 Dans le même "Avant-propos ", Camus défend l'unité des peuples et justifie sa neutralité dans le conflit, condamnant aussi bien la politique colonialiste française de répression que le terrorisme : 
Il semble que la métropole n'ait point su trouver d'autres politiques que celles qui consistaient à dire aux Français d'Algérie : ‘Crevez, vous l'avez bien mérité', ou : 'Crevez-les. Ils l'ont bien mérité'. Cela fait deux politiques différentes, et une seule démission, là où il ne s'agit pas de crever séparément, mais de vivre ensemble.

(Camus, 2000 : 895-96)

"Vivre ensemble» est une unité défendue par Camus qui, toutefois suppose une contradiction : l'ère du colonialisme doit prendre fin, mais l'impérialisme français doit continuer (Camus, 2000 : 1014-15).

Le Renégat souhaite le royaume universel de ses maitres, mais ses espoirs ont une motivation purement intime: quand tous les hommes seront esclaves, il ne sera plus seul : «Ô mes maîtres, ils vaincront la parole et l'amour, (...) et des foules muettes aux pieds entravés chemineront à mes côtés dans le désert du monde sous le soleil cruel de la vraie foi, je ne serai plus seul » (Camus, 2002 : 1592). En vérité, ce qu'il semble redouter le plus c'est le silence du désert et la solitude : «Que le désert est silencieux ! La nuit déjà et je suis seul (...)» (Camus, 2002: 1593). À la fin de la nouvelle, reconvertit aux valeurs humanitaires du christianisme, lorsqu'il tend la main au Fétiche, en geste de paix et d'amitié, le Renégat croit à un dénouement heureux à sa mésaventure mais ses espoirs son vains; la réponse - «Une poignée de sel emplit la bouche de l'esclave bavard » (Camus, 2002: 1593) - confirme sa solitude et se présente comme la négation de toute possibilité d'entendement, de fraternité, pas même de dialogue.

La solidarité semble, de prime abord, être au cœur de la trame de «Les Muets». Cette nouvelle évoque un court épisode de la vie d'Yvars et des ouvriers d'une tonnellerie après une grève qui n'a pas abouti. Ils reprennent le travail mais frustrés. Se sentant humiliés et impuissants, ils gardent le silence face à M. Lassalle, leur patron; silence qu'ils ne rompent pas, même en apprenant la mort de sa fille. Unis dans la colère (Camus, 2002 : 1599), malgré l'apparente bienveillance que Lassalle semble leur porter (Camus, 2002 : 1603), ils se montrent incapables d'exprimer leur solidarité devant le malheur qui le frappe.

Entre les ouvriers, il existe une solidarité et même une certaine fraternité dans la misère - Camus ne distingue pas toujours ces deux notions, parlant de la nécessité d'entente fraternelle entre colons et autochtones -, nommément lors du partage d'un maigre repas et de café, gestes de communion laïque à laquelle ils associent Saïd, le seul Arabe qui travaille dans la tonnellerie (Camus, 2002: 1605). Ce sont, il est vrai, des gestes symboliques de solidarité entre frères d'armes et entre races, mais cette solidarité ne semble, toutefois, retirer sa force que dans l'adversité représentée par M. Lassalle et, de fait, elle n'affaiblit que provisoirement leur sentiment d'impuissance. En vérité, l'intention de Camus, selon Quilliot était de " souligner la solidarité des hommes, que tout ailleurs sépare, devant la mort" (Quilliot, 2002: 2046); solidarité non exprimée verbalement par les ouvriers mais qu'on devine présente dans leur mutisme.

C'est, sans nul doute, dans la nouvelle "L'Hôte », nommément dans l'ethos du héros camusien, Daru, que l'ambigüité entre solitaire et solidaire est le plus fortement présente.

Dans l'univers fictionnel de Camus, cette nouvelle est l'approche la plus directe de la situation politique en Algérie. Selon Quilliot, celle-ci était en projet depuis 1952, avant les événements de Novembre 1954 (Quilliot, 2002: 2048), mais l'évolution politique en Algérie, nommément la révolte latente, dont Camus était pleinement conscient, était trop préoccupante pour ne pas laisser de traces dans cette fiction. 
avec nous $"$ (Camus, 2002:1619), que ce soit une avec nous » (Camus, 2002 : 1619), que ce soit une invitation individuelle ou une invitation à se joindre " eux ", à ses frères rebelles, est un appel à la fraternité et à la solidarité que Daru ne concrétise pas, considérant que "dans les circonstances présentes", les sentiments fraternels sont «des bêtises» qui affaiblissent les hommes (Camus, 2002: 1620). 
31 Ne répondant pas à cet appel pour des raisons personnelles, éthiques et politiques, Daru reste seul. Il découvre que la solitude, condition autrefois nécessaire, dans une relation avec la nature, pour se connaître, a fait place à un état plus complexe, proche de l'exil total ; situation soulignée par la dernière phrase du récit : «Dans ce vaste pays qu'il avait tant aimé, il était seul» (Camus, 2002 : 1623) qui exprime la nostalgie d'un passé sans retour, la tristesse de la constatation de l'isolement que lui impose sa neutralité. Pour lui, l'ultime dilemme de Jonas entre «solidaire » ou "solitaire" (Camus, 2002 : 1654) ne se pose plus. Ironiquement, subvertissant les mots de Camus, on pourrait ne plus dire : «(..) Daru (...) était né [dans ce] pays. Partout ailleurs, il se sentait exilé » (Camus, 2002 : 1612-13), mais : dans ce pays aussi, « il se sentait exilé ».

L'exil est également le sentiment qui afflige D’Arrast, le personnage de «La Pierre qui pousse ". L'action de cette nouvelle se situe au Brésil, plus précisément à Iguape, dans la forêt tropicale où cet ingénieur doit construire une digue pour sauver la population indigène des inondations. Il est accompagné de Socrate, son interprète avec qui il fraternise, et il fait la connaissance d'un indien, le Coq, avec qui il se lie d'amitié.

De prime abord, D'Arrast se sent étranger dans un " continent aux coutumes barbares ", tant face à la population indienne, pauvre mais digne, qu'aux « notables » de la ville, mais aussi étranger à l'Europe mercantile. Après avoir assisté à une Macumba, malgré une certaine fascination, il constate : « Là-bas, en Europe, c'était la honte et la colère. Ici, l'exil ou la solitude, au milieu de ces fous languissants et trépidants, qui dansaient pour mourir» (Camus, 2002: 1678). À Socrate qui lui demande s'il aime la cérémonie, il déclare : «(...), je n'ai pas trouvé ma place, Alors je suis parti.» Et quand celui l'invite : « Reste avec nous, monsieur d'Arrast, je t'aime ». Il lui répond « je voudrais bien, Socrate, mais je ne sais pas danser » (Camus, 2002 : 1679). D'Arrast se sent inhibé, aliéné, face à cette altérité qui l'attire et l'écœure à la fois (Camus, 2002 : 1682). S'il recherche le contact des hommes, cette inhibition l'empêche, dans un premier temps, de connaître une solidarité complète, la véritable amitié. En effet, la relation avec Socrate, bien qu'empreinte de chaleur et même de fraternité - le rire qu'ils partagent en est un signe évident - s'avère superficielle, comme l'est également la compagnie des notables, et en tout cas ne suffit pas à D'Arrast qui cherche un véritable contact avec la population qu'il est venu aider.

La population pauvre d'Iguape se montre naturellement curieuse, sans animosité envers D'Arrast, - le refus, lors de sa première visite, du frère du Coq de lui montrer sa case ne serait qu'un signe de sa dignité dans la pauvreté - et c'est cette curiosité qui pousse le Coq à engager la conversation avec lui. Rudimentaire au départ, la conversation entre les deux hommes s'engage à un niveau autre que celui des dialogues avec Socrate. En effet, leur entente semble s'affirmer et, passant de vérités premières sur la politique et la société, leur conversation s'oriente vers les valeurs spirituelles. Le Coq lui raconte alors sa mésaventure en mer et sa promesse à Jesus de porter une pierre jusqu'à l'église et, finalement, demande à D'Arrast s'il n'a jamais « appelé » :

'Et toi, n'as-tu jamais appelé, fait une promesse?

- Si, une fois, je crois.

- Dans un naufrage?

- Si tu veux' Et d'Arrast dégagea sa main brusquement. Mais au moment de tourner

les talons, il rencontra le regard du coq. Il hésita, puis sourit.

'Je puis te le dire, bien que ce soit sans importance Que1qu'un allait mourir par ma faute.

Il me semble que j'ai appelé. 
- Tu as promis?

- Non. J'aurais voulu promettre.

- Il y a longtemps?

- Peu avant de venir ici.'

Le coq prit sa barbe à deux mains. Ses yeux brillaient. Tu es un capitaine, dit-il. Ma

maison est la tienne. Et puis tu vas m'aider à tenir ma promesse, c'est comme si tu

la faisais toi-même. Ça t'aidera aussi.'

D'Arrast sourit : 'Je ne crois pas.'

- Tu es fier, capitaine.

- J'étais fier, maintenant je suis seul. (Camus, $2002: 1672$ ) profonde et on assiste ainsi à la naissance d'une fraternité qui, toutefois, ne sera pas effective dès cet épisode, ni même après le partage du dîner dans la case du frère du Coq. De fait, cette amitié ne se concrétisera que le lendemain, avec la solidarité de D'Arrast envers le coq : sous le poids de la pierre, le Coq s'effondre, D'Arrast qui, comme promis, l'accompagnait, charge la pierre sur ses épaules mais au lieu de la porter à l'église, il se dirige vers la case du Coq et la jette au « centre de la pièce » (Camus, $2002: 1685)$. Ce geste peut être passible de diverses interprétations : refus des croyances religieuses, voire des vestiges coloniaux, mais elle est, selon nous, le symbole d'une amitié, une nouvelle pierre qui pousse :

D'Arrast, debout dans l'ombre, écoutait, sans rien voir, et le bruit des eaux l'emplissait d'un bonheur tumultueux. Les yeux fermés, il saluait joyeusement sa propre force, il saluait, une fois de plus, la vie qui recommençait. Au même instant, une détonation éclata qui semblait toute proche. Le frère s'écarta un peu du coq et se tournant à demi vers d'Arrast, sans le regarder, lui montra la place vide : 'Assieds-toi avec nous.' (Camus, 2002 : 1686)

En aidant le Coq à tenir sa promesse, même s'il l'a déniée en ne portant pas la pierre à l'église, D'Arrast, comme l'avait prédit le Coq, a été libéré d'un fardeau qu'il ne s'avouait qu'indirectement. Comme Janine sur la terrasse du fort, il retrouve un bonheur intérieur et une harmonie longtemps oubliés: «(...) il écouta monter en lui le flot d'une joie obscure et haletante qu'il ne pouvait pas nommer » (Camus, 2002: 1685). D'Arrast est le seul personnage camusien de L'Exil et le Royaume et, sans doute, de toute l'œuvre camusienne publiée de son vivant qui, à travers la solidarité, connaît la communion avec l' « autre » et atteint le bonheur dans la fraternité ${ }^{5}$.

Ainsi, dans L'Exil et le Royaume, Camus nous offre diverses tonalités de la relation à l'autre, du difficile équilibre entre être solidaire ou solitaire. Selon Haouet, une grande partie des personnages camusiens recherche la solidarité mais souffre de solitude (Haouet, 2003: 94), Pour Jonas, cet équilibre est encore possible, pour Yvars la solidarité semble éphémère, mais Janine, le Renégat, et Daru, tant pour des motifs particuliers que politiques, semblent être définitivement condamnés à la solitude. D’Arrast dans « La Pierre qui pousse", semble faire exception. Cette nouvelle serait une touche finale lumineuse d'un tableau manifestement obscur en ce qui concerne les relations humaines. 


\section{BIBLIOGRAPHIE}

CAmus, Albert (2000). Essais. Paris : Gallimard, « Bibliothèque de la Pléiade ».

CAmus, Albert (2002). L'Exil et le Royaume, in Albert Camus. Théâtre, Récits, Nouvelles. Paris :

Gallimard, «Bibliothèque de la Pléiade », pp. 1553- 1686.

CRYLE, Peter (1973). Bilan Critique : L'Exil et le Royaume d'Albert Camus, essai d'analyse. Paris : Minard.

FiтcH, Brian, T. (1964). Le sentiment d'étrangeté chez Malraux, Sartre, Camus et S. de Beauvoir :

"étranger à moi-même et à ce monde ». Paris : Minard.

FiтcH, Brian, T. (1988). «'La Femme adultère' : A Microcosm of Camus' Solipsistic Universe », in

Anthony Rizzuto (éd.). Albert Camus L'Exil et le Royaume, The Third Decade. Toronto : Paratexte, pp. 117- 26.

GASSIN, Jean (1981). L'univers symbolique d'Albert Camus, essai d'interprétation psychanalytique. Paris : Minard.

GAY-CROSIER, Raymond (1988). « Renegades Revisited », in Anthony Rizzuto (éd.). Albert Camus' L'Exil et le Royaume, The Third Decade. Toronto : Paratexte, 1988, pp. 19- 33.

GrouIX, Pierre (2009). « Exil et le Royaume (L') », in Jeanyves Guérin (dir.). Dictionnaire Albert Camus. Paris : Robert-Lafont, pp. 301-06.

HAQUET, Mohamed Kamel Eddine (2003). Camus et l'hospitalité. Paris : L'Harmattan.

MAILHOT, Laurent (1973). Albert Camus ou L'Imagination du désert. Montréal : Les Presses de l’Université de Montréal.

Nicolas, André (1964). Albert Camus : Une philosophie de l'existence. Paris : Presses Universitaires de France.

Quillot, Roger (2002). « Commentaires, notes et variantes », in Albert Camus. Théâtre, récits, nouvelles. Paris : Gallimard, « Bibliothèque de la Pléiade », pp. 1687-2070.

Quillot, Roger (2000). « Commentaires », in Albert Camus. Essais. Paris : Gallimard, « Bibliothèque de la Pléiade », pp. 1839-1847.

SAID, Edward W. (1979). Orientalism. New York : Vintage Books.

\section{NOTES}

1. Pour Jean Gassin, dans l'œuvre de Camus, la Pierre symbolise, dans les premiers textes, la chaleur de la vie. Toutefois, cet élément acquiert progressivement des aspects négatifs symbolisant le destin individuel, la mort qui attend et fascine tout homme. Ce symbolisme atteint une dimension cosmique vu que l'indifférence de la pierre nous renvoie à l'indifférence du monde et, de la confrontation de l'homme avec le monde nait l'absurde (Gassin, 1981 : 43-50). Laurent Mailhot consacre tout un chapitre - «La Pierre » - à cet élément, en particulier au rocher de Sisyphe (Mailhot, 1973 : 229-53). 
2. Imaginaire transmis par un ensemble de figurations littéraires entre autres, sujettes à des transformations populaires - «a kind of free-floating mythology of the Orient» -, comme l'établit Edward Said dans Orientalism (1979: 52-3).

3. Vd. l'analyse de Gassin sur la duplicité du symbolisme de la nuit dans le chapitre "Nuit » (Gassin, 1981 : 51-64).

4. On utilise les guillemets à « autre " pour référer au sujet colonisé.

5. Soulignons que cette rencontre avec l'autre se situe dans la forêt amazonienne, loin de l'adversité politique qui fustige l'Algérie et entrave l'amitié entre Daru et le prisonnier.

\section{RÉSUMÉS}

Parcourant les nouvelles de L'Exil et le Royaume, on montre que les héros camusiens sont tous confrontés au dilemme "solitaire ou solidaire ", qu'ils aspirent à la communication avec l'autre et le monde, bref, au royaume, mais se trouvent face à un triple isolement - géographique, politique et psychologique - qui s'avère être l'essence de leur exil. Toutefois, on constate que, parce que placé dans un contexte géopolitique différent - l'Amérique du sud et non pas L'Algérie d'avant-guerre - le protagoniste de "La Pierre qui pousse» échappe à cette fatalité, que l'isolement et la solitude peuvent mener à la solidarité et même à la fraternité.

Reading the short stories of L'Exil et le Royaume, we show that Camusian heroes are all faced with the "solitaire ou solidaire" dilemma, that they aspire to communicate with the other and the world, in short, to the kingdom, but face a triple isolation - geographical, political and psychological - which proves to be the essence of their exile. However, we find that, because he is placed in a different geopolitical context - South America and not the pre-war Algeria - the protagonist of "La Pierre qui pousse" escapes this fate, the isolation and loneliness that can lead to solidarity and even brotherhood.

\section{INDEX}

Keywords : Camus (Albert), isolation, solitary, solidary, exile

Mots-clés : Camus (Albert), isolement, solitaire, solidaire, exil

\section{AUTEUR}

FERNANDO GOMES

Un. d'Évora - CEL - Centro de Estudos em Letras

fgomes[at]uevora.pt 